\title{
Evaluation of Esterification and Membrane Based Solvent Extraction as Methods for the Recovery of Short Chain Volatile Fatty Acids from Slaughterhouse Blood Anaerobic Mixed Fermentation
}

\author{
Jersson Plácido $^{1,2}$ [ $\cdot$ Yue Zhang ${ }^{1}$
}

Received: 24 October 2016 / Accepted: 6 April 2017 / Published online: 11 May 2017

(C) The Author(s) 2017. This article is an open access publication

\begin{abstract}
Two routes, esterification and membrane based solvent extraction, were tested for their efficiency to extract volatile fatty acids (VFA) (acetic, propionic, butyric, isobutyric, iso-valeric and valeric acids) produced from the anaerobic-mixed fermentation of slaughterhouse blood. A range of operational parameters and reagents were assessed to optimize each system. Esterification recovered VFA as methyl esters with the production of ammonium sulphate as a value-added by-product of the esterification reaction. A membrane extraction system, comprised of a hydrophobic membrane and extractant using octanol/TOA (trioctylamine), was efficient to separate butyric and iso-valeric acids in favour of acetic acid from the fermentation broth. These results provided important information for the development of a carboxylate-platform bio-refinery using highprotein wastes as substrate.
\end{abstract}

Keywords Volatile fatty acids $\cdot$ Membrane based solvent extraction · Esterification $\cdot$ Purification $\cdot$ Anaerobic mixed fermentation

\section{Introduction}

The transformation of petroleum-based processes into biological-based processes expects to save 2.5 billion tons of

Jersson Plácido

j.e.placidoescobar@swansea.ac.uk

1 Water and Environmental Engineering Group, Faculty of Engineering and the Environment, University of Southampton, Southampton, UK

2 Medical School, Centre for Cytochrome P450 Biodiversity, Institute of Life Science (ILS 1), Swansea University, Room 529, Swansea SA2 8PP, Wales, UK
$\mathrm{CO}_{2}$ per year by 2030 [1]. Petroleum-based materials and chemical have been replaced by biological-based materials and chemicals with environmental advantages such as sustainability and biodegradability $[2,3]$. One of these petroleum based-chemicals is the short chain carboxylic acids or volatile fatty acids (VFA). VFA are carboxylic acids with a carbon chain between 2 and 7 carbons [acetic (2), propionic (3), iso-butyric (4), $n$-butyric (4), iso-valeric (5), valeric (5), hexanoic (6), and heptanoic (7)]. Industrially, VFA are produced in a petroleum-based process using different types of chemical reactions such as oxidations, dehydrogenation, carbonylation, etc. VFA are utilized in several industries as precursor of different products such as solvents (methyl acetate, ethyl acetate), inks, coatings (cellulose acetate, cellulose butyrate) [4], polymers [cellulose acetate, polyvinyl acetate, polyhydroxialkanoates (PHA)], artificial flavors (ethyl butyrate, ethyl propionate), and scents (methyl butyrate, methyl valerate). The several VFA uses have generated a multibillion dollars industry with a considerably predicted increment in the next years [5].

Besides petroleum-based processes, VFA can be produced by biological methods. Acetic acid production by anaerobic fermentation is the most well-known VFA production. This process is only applied in the vinegar production because it is still economically uncompetitive compared with the acetic acid petroleum-based production. To reduce the difference between petroleum and biological based VFA production it is necessary to improve the upstream and downstream processing. The upstream processes have been improved by using wastes as substrates to produce VFA [6-9]. Carbohydrate based residues such as paper wastes, lignocellulosic material, municipal solid wastes, etc. are the most common wastes employed in the production of VFA. In our past research, we evaluated the production of VFA using protein rich 
substrates [10]; in this work, the Anaerobic mixed fermentation (AMF) of slaughterhouse blood reached concentrations of between 70 and $100 \mathrm{~g} \mathrm{~L}^{-1}$. The principal VFA species were acetic $25-30 \mathrm{~g} \mathrm{~L}^{-1}, n$-butyric 25-30 $\mathrm{g} \mathrm{L}^{-}$, and iso-valeric $15-30 \mathrm{~g} \mathrm{~L}^{-1}$. These results are the largest VFA concentrations produced from agroindustrial wastes. Therefore, the promising upstream process made necessary the development of a VFA downstream methodology to complement the VFA process.

Carboxylic acids production costs are dominated by the feedstock and fermentation costs; however, the downstream processing is around $30-40 \%$ of the production costs [11]. The upstream costs are not significant in the VFA production from slaughterhouse blood; therefore, the principal processing costs are associated with the downstream processing. The recovery of carboxylic acids from fermentation broths is dependent of the process configuration, acid structure, and process economics. The recovery process follows a series of unit processes, e.g. clarification, primary recovery, counterion removal, concentration, upgrading and formulation [12]. VFA purification employs different types of technologies such as adsorption (ion exchange, anion exchange), extraction (liquid-liquid, reactive), precipitation, electrodialysis, and filtration.

Two important characteristic in AMF of slaughterhouse blood are the high ammonia concentration $\left(5-12 \mathrm{~g} \mathrm{~L}^{-1}\right)$ and the fermentation broth's $\mathrm{pH}$ (6.5-8); therefore, the VFA are expected to be present as ammonium carboxylates. Ammonium carboxylates have been purified using advanced precipitation and esterification. In this case, ammonium lactate was transformed into methyl lactate with a yield of $75 \%$ [13]. The advantage of esterification for VFA recovery is the production of VFA-esters which are compounds with wide applications in scents and fragrance industry and ammonium sulphate a well-known fertilizer. Other option for VFA recovery is the application of membrane technologies. Membrane technologies have recovered VFA from different aqueous systems by using methodologies such as nanofiltration, ultrafiltration, pervaporation and pertraction. Membrane based solvent extraction (MBSE) is a promising process that allows the removal of organic compounds from aqueous system into an organic extractant. Generally, the organic extractant is a mixture of an organic solvent and an amine. The use of amines and especially tertiary amines have become in a noticeable option to separate VFA from fermentation broths. Tertiary amines such as trioctylamine have extracted between 60 and $90 \%$ of VFA from complex solutions [14]. In the future, MBSE technology can be utilized as a continuous recovery method for VFA production from agricultural and foods wastes. The competitive production of chemical compounds from AMF and future VFA bio-refineries from high-protein wastes are associated with the advance in recovery technologies such as esterification and MBSE.

The aim of this work was to evaluate esterification and MBSE as two possible methodologies for VFA recovery from aqueous solution and their suitability for the recovery of VFA produced from AMF of slaughterhouse blood.

\section{Materials and Methods}

\section{Materials}

The analytical-grade reagents used in this study, as listed below, were all obtained from Sigma-Aldrich (Dorset, $\mathrm{UK}$ ): Acetic, propionic, iso-butyric, $n$-butyric, iso-valeric, valeric acid, sulphuric acid, methanol, methyl acetate, methyl propionate, methyl butyrate, methyl iso-butyrate, methyl iso-valerate, ammonium hydroxide, trioctylamine (TOA), and octanol.

\section{Fermentation Processes}

To simulate slaughterhouse blood, commercial freeze dried blood for black pudding (Tongmaster Seasonings, Airdrie, UK) was employed. The blood was prepared using one part of blood for six parts of water. The fermentation process was performed using 10\% of inoculum and $90 \%$ of blood solution. Sieved sewage sludge digestate samples from Millbrook wastewater treatment (Southampton, United Kingdom) were utilized as inoculum. The culture was fermented using a batch and semi-continuous configurations. Batch cultures were performed during 10 days achieving a production of $100 \mathrm{~g} \mathrm{~L}^{-1}$ and a VFA profile with acetic (30\%), butyric (30\%), iso-valeric (20\%) iso-butyric $(15 \%)$ and propionic (5\%) acids. On the other hand semi-continuous culture with a SRT of 7 days achieved an average production of $70 \mathrm{~g} \mathrm{~L}^{-1}$ and a similar VFA profile as the batch culture. Both cultures were kept at $37^{\circ} \mathrm{C}$ and $150 \mathrm{rpm}$.

\section{Esterification}

Synthetic model VFA solutions and real slaughterhouse blood fermentation broth were used to evaluate the esterification approach.

\section{Esterification of Synthetic Model VFA Solutions}

Model solution experiments were performed under a factorial design $2 \times 2$, three replicates. The model solution experiment evaluated the effect of VFA: methanol $(\mathrm{CH} 3 \mathrm{OH})$ ratio $(1: 1$ and 1:0.5 on volume basis) and $\mathrm{pH}$ for esterification reaction (4.5 and 6.5). In all this experiment, the proportion of VFA to sulphuric acid $\left(\mathrm{H}_{2} \mathrm{SO}_{4}\right)$ 
was kept at the same volume ratio (1:0.3 on volume basis). The initial experiment was performed using a $50 \%$ model VFA with a VFA profile similar to the observed in the batch fermentation of slaughterhouse blood [acetic (30\%), butyric (30\%), iso-valeric (20\%) iso-butyric (15\%) and propionic (5\%)]. Ammonium carboxylates were produced by mixing the acids solution with a $5 \mathrm{~N}$ solution of ammonium hydroxide until the solution achieved a $\mathrm{pH}$ comparable to the slaughterhouse blood fermentation broth (6.5-8). To evaluate the esterification approach to those ammonia carboxylate solutions, the ammonium carboxylates were acidified by adding sulphuric acid $\left(\mathrm{H}_{2} \mathrm{SO}_{4}\right)$ into the ammonium carboxylates solution, it was applied as the first step to create the optimal $\mathrm{pH}$ for esterification reaction. After that, certain amount of methanol $\left(\mathrm{CH}_{3} \mathrm{OH}\right)$ was added to the solution and the mixture was then agitated at $20^{\circ} \mathrm{C}$ and $150 \mathrm{rpm}$ for $2 \mathrm{~h}$ in order for methanol reacting with the VFA to produce methyl esters. A significant amount of precipitation, confirmed to be ammonium sulphate $\left[\left(\mathrm{NH}_{4}\right)_{2} \mathrm{SO}_{4}\right]$ salt, was also resulted in this process. After the designated reaction time, the solutions were centrifuged for $30 \mathrm{~min}$ at $5000 \mathrm{rpm}$ (Sorvall Legend XTR Centrifuge, Fisher Scientific Ltd, UK) to separate solid phase (ammonium sulphate), water phase, and ester phase. Samples for each liquid phase were stored in a freezer at $-20^{\circ} \mathrm{C}$ before analyses, and the solids were dried overnight at $105^{\circ} \mathrm{C}$ for analysis.

\section{Esterification of Slaughterhouse Blood Fermentation Broth}

The fermentation broth assay evaluated VFA concentration $[50$ and $80 \%,(\mathrm{w} / \mathrm{w})]$ and $\mathrm{VFA}: \mathrm{H}_{2} \mathrm{SO}_{4}: \mathrm{CH}_{3} \mathrm{OH}$ ratio (1:0.3:0.5, 1:0.3:1, 1:0.5:0.5, 1:0.5:1 on a molar basis) using a factorial design $2 \times 4$ and three replicates. Ammonium sulphate yield and methyl VFA yield were used as response variables. The esterification methodology applied for the fermentation broth had a different process from the model solutions. When the fermentation process finished, the fermentation broth was centrifuged for $20 \mathrm{~min}$ at $5000 \mathrm{rpm}$ (Sorvall Legend XTR Centrifuge, Fisher Scientific Ltd, UK) and the supernatant was taken for esterification. To achieve the same concentration of the model solutions, the supernatant was rotovaporated (Buchi Brinkmann Re-111 Rotavapor. Buchi, UK) until achieve a volume reduction between 50 and $80 \%$ in evaporation flask. Samples from the distillate and residue were taken for VFA analyses. The heavy phase/residue was collected and the same process performed for the model solutions was performed for the VFA-laden heavy phase. The best treatment was selected and used to evaluate the reaction kinetic and to evaluate the temperature effect $\left(25-60^{\circ} \mathrm{C}\right)$ in the esterification reaction.
Membrane Based Solvent Extraction (MBSE) System

\section{Octanol/TOA Ratio Optimization}

To optimize the ratio of octanol and TOA, a set of batch experiments using 10\% VFA model solution was performed. The experiment utilized a factorial design $2 \times 3$ using the TOA concentration in the octanol/TOA solution (10 and 20\%) and the feed/solvent (VFA to Octanol/ TOA) ratios $(1: 1,2: 1$, and $4: 1)$ as factors. This experiment was performed in $50 \mathrm{~mL}$ centrifuge flask with three replicates at room temperature and $150 \mathrm{rpm}$ during $2 \mathrm{~h}$. After $2 \mathrm{~h}$, the samples were centrifuged for $20 \mathrm{~min}$ at 5000 rpm (Sorvall Legend XTR Centrifuge, Fisher Scientific Ltd, UK) and samples from the aqueous phase were analysed. The amount of recovered VFA was calculated using Eq. 1:

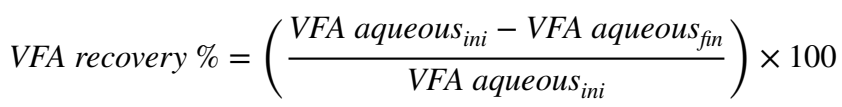

The treatment that achieved the best VFA recovery was selected as organic solvent in the MBSE system.

\section{Membrane Based Solvent Extraction Experiments}

The MBSE system employed in this experiment consisted of a hollow fibre hydrophobic membrane module $\left(0.5 \times 1\right.$ Micromodule ${ }^{\circledR}$ membrane contactor, Membrana, North Carolina, USA). The system's diagram is showed in Fig. 1. In the outside of the membrane VFA model solutions or real fermentation broth was transported, whereas, inside the membrane a solution of octanol/TOA was pumped. All the tubing was solvent and acid resistant to avoid any damage caused by the liquids utilized in the experiment. Before starting the MBSE process the VFA and TOA/octanol solutions were filtered using a Whatman filtration apparatus with 0.45 (nylon Whatman) and $0.22 \mu \mathrm{m}$ (PTFE filters) filters. The fermentation broth was centrifuged at $5000 \mathrm{rpm}$ (Sorvall Legend XTR Centrifuge, Fisher Scientific Ltd, UK) for $1 \mathrm{~h}$ and filtrated using the $0.45 \mu \mathrm{m}$ filters. The system was warmed up using the lower flow allowed by the pumps. After warm up, the MBSE system used a flow of $2 \mathrm{~mL} \mathrm{~min}^{-1}$ for the internal circuit and $15 \mathrm{~mL} \mathrm{~min}^{-1}$ for the external circuit. The recovery was performed continuously for $2 \mathrm{~h}$ and samples were taken each half an hour. Additionally, a kinetic study was performed for $24 \mathrm{~h}$ using the fermentation broth. The amount of recovered VFA was calculated using Eq. 1. 
Fig. 1 Schematic diagram of the MBSE system utilized for VFA recovery

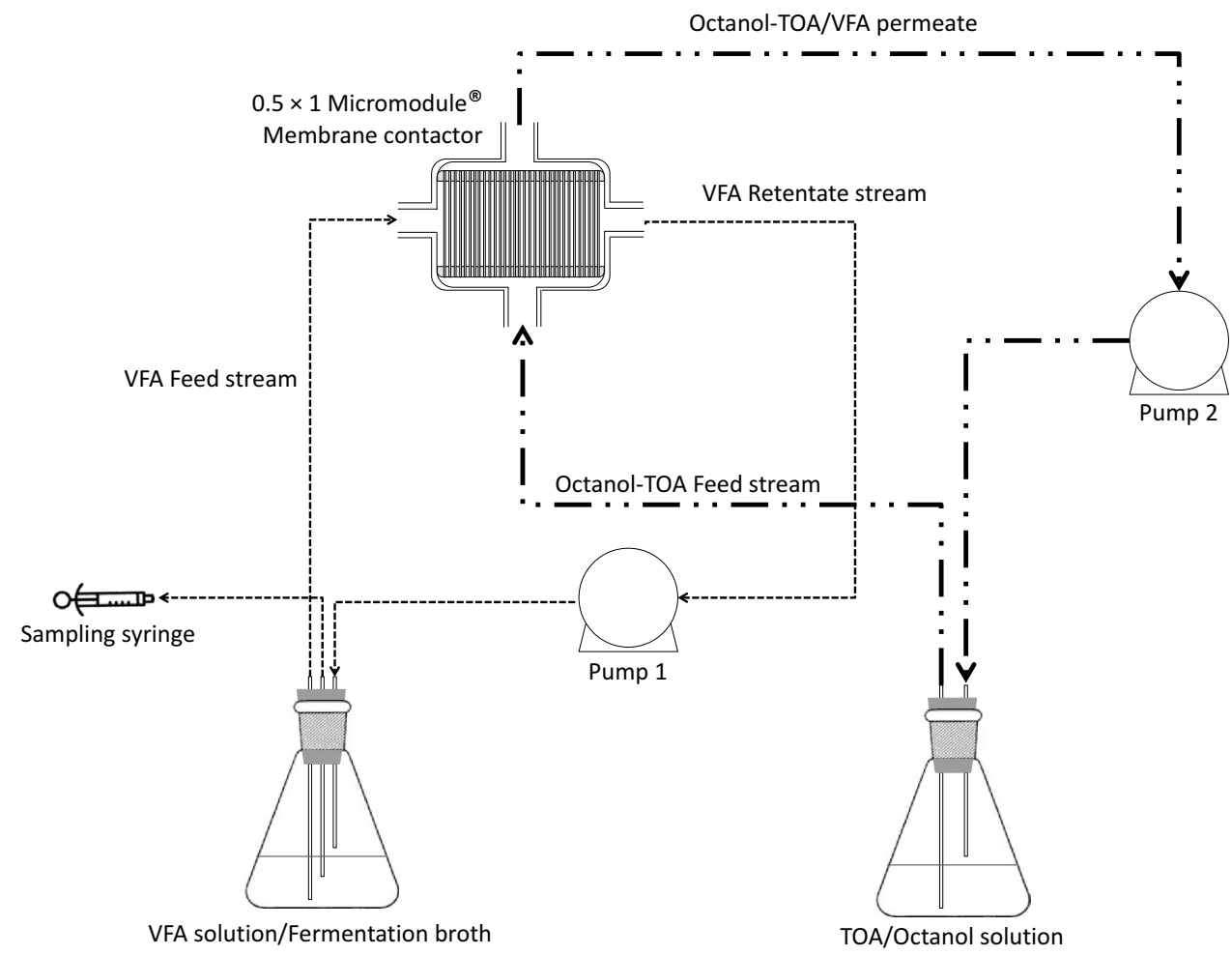

\section{Analytical Methods}

The quantification of VFA and methyl esters was performed by a Shimadzu GC-2010 gas chromatograph (Shimadzu, Milton Keynes, UK), using a flame ionization detector and a capillary column type SGE BP-21. Samples were prepared for analysis by centrifugation at $14,000 \mathrm{~g}$ (micro-centrifuge, various manufacturers) for $20 \mathrm{~min}$. Where dilution was necessary, deionized water was supplemented and formic acid was then added to give a concentration of $10 \%$ of the total volume for analysis. The acidified solution was transferred into the vials and loaded onto the GC auto-sampler. The GC oven temperature was programmed to increase from 60 to $210^{\circ} \mathrm{C}$ in $15 \mathrm{~min}$ with a final hold time of $5 \mathrm{~min}$.

The amount of ammonium sulphate produced during esterification was quantified by the barium chloride method [15].

\section{Results}

\section{Esterification of Synthetic Model VFA Solutions}

VFA model solutions were utilized to obtain the basic conditions for producing VFA methyl esters and $\left(\mathrm{NH}_{4}\right)_{2} \mathrm{SO}_{4}$. The experiment conditions evaluated the effect of two $\mathrm{pH}$ conditions and the VFA:Methanol proportion. In all this (a)

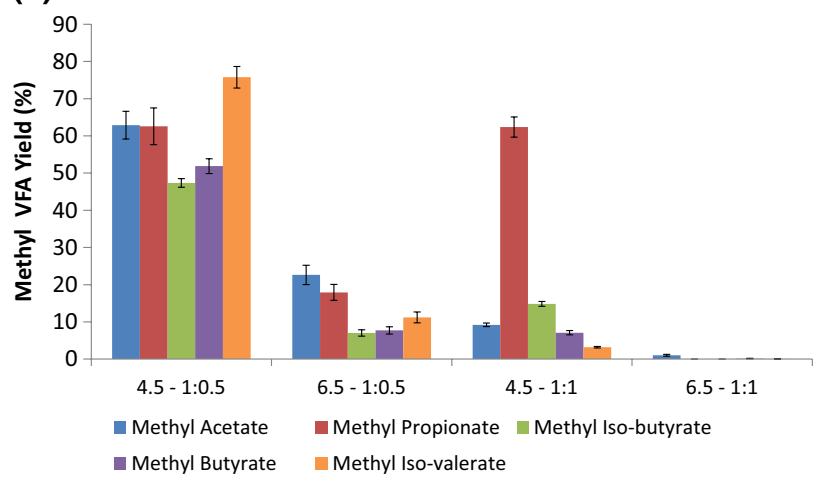

(b)

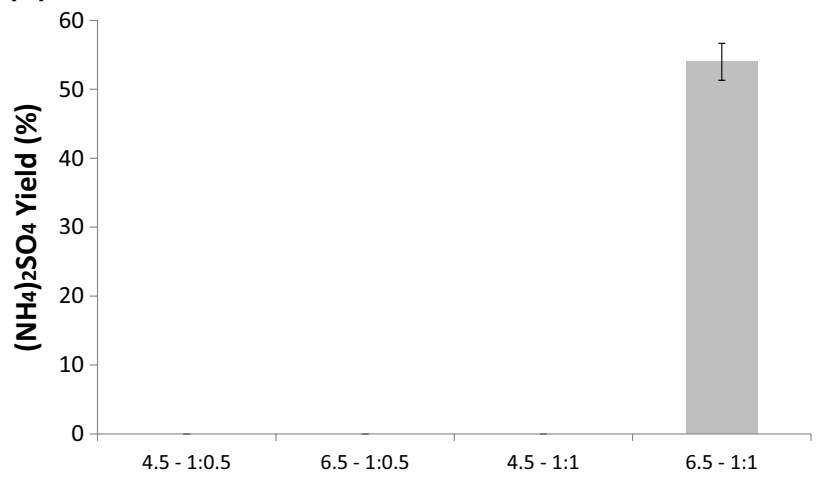

Fig. 2 Bar plots for the yields obtained by the esterification reaction of model VFA solution. a Mehtyl VFA yield (\%), b $\left(\mathrm{NH}_{4}\right)_{2} \mathrm{SO}_{4}$ Yield (\%) 
experiment, the proportion of VFA to sulphuric acid was kept at the same volume ratio (1:0.3). Figure 2 shows the yield obtained for the production of methyl VFA (Fig. 2a) and $\left(\mathrm{NH}_{4}\right)_{2} \mathrm{SO}_{4}$ (Fig. 2b). The treatment that produced the largest methyl VFA yields had acid $\mathrm{pH}$ (4.5) and a 1:0.5 VFA: $\mathrm{CH}_{3} \mathrm{OH}$ relation. This treatment achieved a methyl esters yield above $45 \%$ for the five acids evaluated; the methyl ester with the highest yield was methyl iso-valerate (75\%), followed by methyl acetate $(62 \%)$ and methyl propionate $(62 \%)$. The other treatments produced considerably less amounts of methyl esters. The treatments with lower $\mathrm{pH}$ generated a larger amount of methyl esters compared with the ones with neutral $\mathrm{pH}$. Neutral $\mathrm{pH}$ affected the esterification reaction in both VFA: $\mathrm{CH}_{3} \mathrm{OH}$ ratios; however, the $\mathrm{pH}$ effect was greater in the 1:1 VFA: $\mathrm{CH}_{3} \mathrm{OH}$ ratio. The 1:0.5 ratio obtained greater methyl esters yields than the 1:1 except for the methyl propionate that achieved similar yields in both ratios.

The $\left(\mathrm{NH}_{4}\right)_{2} \mathrm{SO}_{4}$ yield (Fig. 2b) exhibited a different behaviour compared with the methyl esters yield. The only treatment that produced ammonium sulphate was the 6.5-1:1, this treatment achieved a production yield of $54 \%$, however, this treatment generated the lowest amount of methyl esters among the treatments evaluated. In this treatment, the larger amount of methanol mixed with a non-acidic $\mathrm{pH}$ favoured the reaction between the ammonium and the $\mathrm{H}_{2} \mathrm{SO}_{4}$ instead of favour the reaction between VFA and methanol. The treatments that produced methyl VFA in considerable amounts generated two liquid phases, whereas, the treatment that produced ammonium sulphate as the principal product did not exhibited two liquid phases. Nevertheless, the results obtained from the model solutions proved that a mixture of VFA and ammonium ions can react with methanol and sulphuric acid for the production of methyl VFA and ammonium sulphate.

\section{Esterification of Slaughterhouse Blood Fermentation Broth}

Although the esterification reaction between solutions with ammonium and VFA and methanol was proved, the conditions of this reaction can varied when a complex solution as a fermentation broth is utilized as a part of the reaction. Therefore, to improve the methyl esters and ammonium sulphate precipitation, the esterification reaction using fermentation broth evaluated two levels of water reduction and different relations among VFA, $\mathrm{H}_{2} \mathrm{SO}_{4}$ and $\mathrm{CH}_{3} \mathrm{OH}$. Figure 3 shows the yields obtained by the esterification of VFA from slaughterhouse fermentation. The best production of methyl VFA was obtained using 80\%-1:0.3:0.5 (Fig. 3a), this treatment produced a VFA yield between 35 and $50 \%$, the largest yield was obtained by propionic and iso-valeric acid. In fact, this
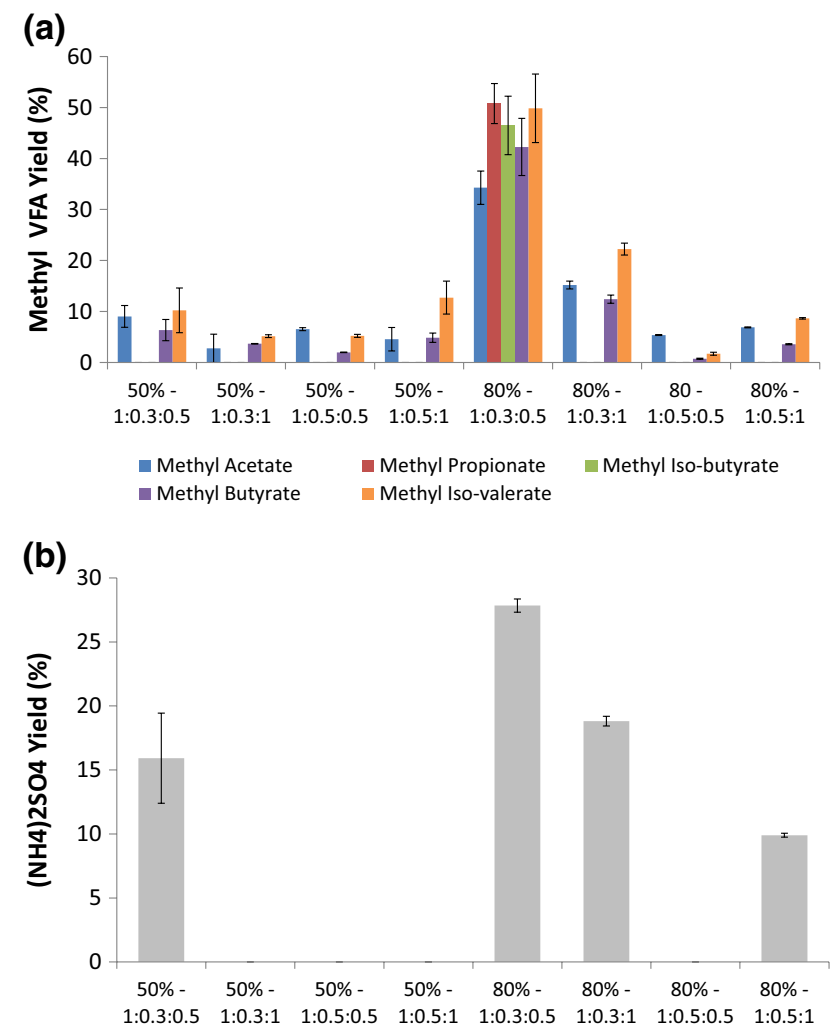

Fig. 3 Esterification reaction yield for the VFA produced from the slaughterhouse blood mixed fermentation broth. a Mehtyl VFA yield $(\%), \mathbf{b}\left(\mathrm{NH}_{4}\right)_{2} \mathrm{SO}_{4}$ yield (\%)

was the only treatment that generated a non-polar phase. Additionally, The 80\%-1:0.3:0.5 treatment was the only condition that produced methyl propionate and methyl iso-butyrate. The treatments with $80 \%$ VFA concentration increased the production of methyl esters compared with the $50 \%$ treatments, this increment is principally due to the increment in the production of methyl acetate, which increased in almost twofold between the treatments with 50 and $80 \%$ VFA. The 1:0.5:0.5 ratio produced the lowest methyl ester yield in both VFA concentrations, in those treatments only were observed methyl acetate, methyl butyrate, and methyl iso-valerate and all of them in concentrations below $10 \%$. In general, the yield obtained by the fermentation broth esterification was lower than the one obtained by the model solution (Fig. 2a). The acid that exhibited the largest reduction in its transformation was acetic acid with a reduction close to $30 \%$; in contrast, butyric and iso-butyric showed almost the same yield than the model solutions esterification.

Different from the model solutions, the fermentation broth did not produce a precipitate solely containing ammonium sulphate. When a blackish solid phase was produced from the esterification of the fermentation 
broth, the amount of ammonium sulphate in the solids corresponded to $50 \%$ of the solid phase produced from the esterification reaction. The additional materials were suspected to be fermentation residues that were aggregated or oxidized by the addition of sulphuric acid. Likewise the methyl esters yield, the largest production of ammonium sulphate was performed by the treatment 80\%-1:0.3:0.5 (Fig. 3b), this treatment achieved an ammonium sulphate yield of $28 \%$ followed by the treatments $80 \%-1: 0.3: 1(19 \%), 80 \%-1: 0.5: 1(10 \%)$ and 50\%-1:0.3:0.5 (16\%). The other treatments did not produce any solid phase during the esterification reaction. As the treatment 80\%-1:0.3:0.5 produced the largest yields of methyl esters and ammonium sulphate this treatment was selected for further experiments.

The fermentation broth esterification reaction kinetic was performed during $24 \mathrm{~h}$ (Fig. 4a). All methyl esters achieved the highest production after $24 \mathrm{~h}$, those yields varied from 38 to $49 \%$. During the first $2 \mathrm{~h}$ the esterification yields increased linearly and reached an equilibrium position after $8 \mathrm{~h}$. The highest yield was obtained by methyl iso-valerate (49\%), methyl iso-butyrate (48\%) and methyl propionate $(45 \%)$. Similar to the past experiment methyl acetate reached the smallest yield (38\%). At $2 \mathrm{~h}$ the methyl
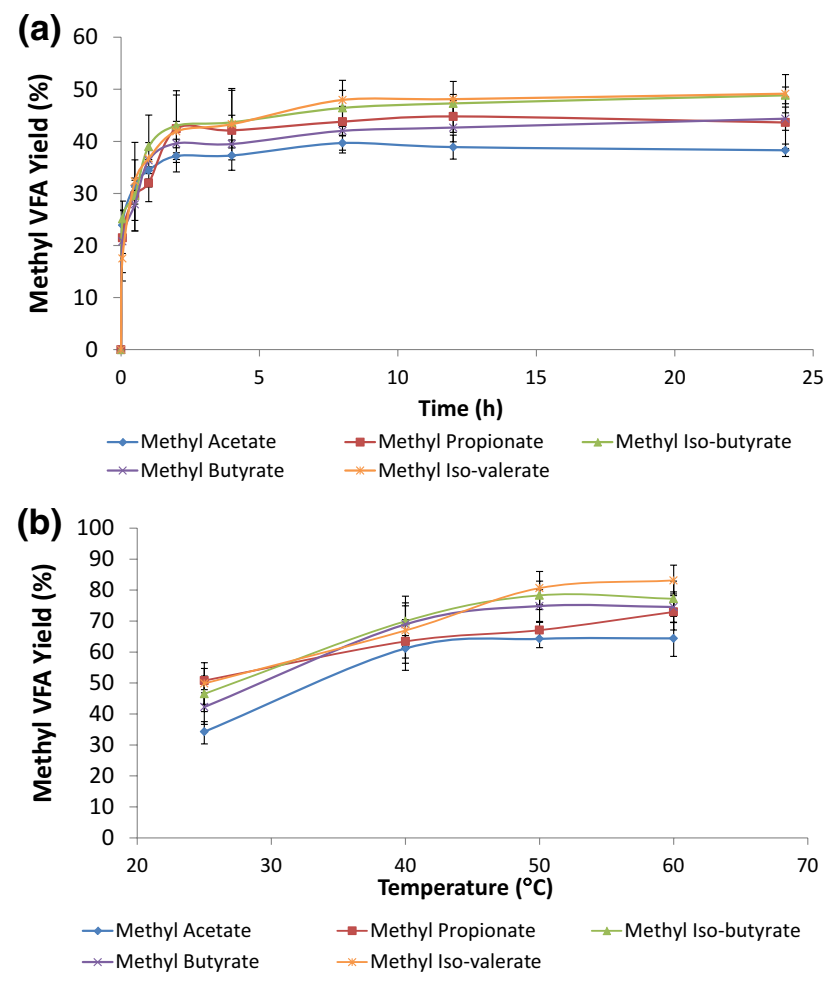

Fig. 4 a Reaction kinetic for the FA produced from the slaughterhouse blood mixed fermentation broth. b Esterification reaction temperature for the VFA produced from the slaughterhouse blood mixed fermentation broth
VFA reached between 90 and $97 \%$ of their maximum yield. This indicates that $2 \mathrm{~h}$ is a reasonable time to stop the reaction without conceding a significant loss of products and allowing a reduction in energy costs.

To improve the esterification yield different temperatures were evaluated $\left(40,50\right.$ and $\left.60^{\circ} \mathrm{C}\right)$. The esterification yields obtained under different temperatures is shown in Fig. 4b. The acids esterification yields increased with the temperature raise. The esterification yield increased approximately $30 \%$ when compared with the initial experiments. The greatest esterification yields were obtained at $60^{\circ} \mathrm{C}$, at this temperature the esterification yield achieved yields between 65 and $84 \%$. However, the difference between 50 and $60^{\circ} \mathrm{C}$ was not significant. Comparable to the other esterification experiments, the highest transformation occurred in the methyl iso-valerate and the lowest one in methyl acetate. The majority of methyl esters achieved their best production at $60^{\circ} \mathrm{C}$; however, the difference between 50 and $60^{\circ} \mathrm{C}$ was not significant to set the reaction temperature at $60^{\circ} \mathrm{C}$; therefore, in future studies $50^{\circ} \mathrm{C}$ will be used as the reaction temperature. On the other hand, the ammonium sulphate yield was not affected by the changes in the temperature and it was stable at the same values as the past fermentation broth experiment (50\%).

\section{Membrane Based Solvent Extraction (MBSE) System}

\section{Octanol/TOA Ratio Optimization}

One of the initial steps to implement a MBSE system for the recovery of VFA from aqueous solution is the selection of the solvent to be utilized inside the hollow fibre membranes. Trioctylamine (TOA) and octanol were selected as the extractant pair in the MBSE system. This selection was based in previous researchers in the extraction of carboxylic acids from aqueous solution using liquid-liquid extraction [16-19]. The results of the selection experiment for the recovery of VFA model solutions [10\% solution of VFA with a composition of acetic (30\%), butyric (30\%), iso-valeric (20\%), iso-butyric (15\%) and propionic acids (5\%)] using different TOA concentrations and VFA to TOA/octanol solution are shown in Fig. 5a. All the treatments were able to recover VFA from the model solution mixture. The total VFA recovery efficiency varied from 49 to $77 \%$. In terms of total VFA recovery, the treatments with a 1:1 VFA:TOA/octanol obtained the best results (77 and $74 \%$ ) followed by the 2:1 (67 and 62\%) and 4:1 (53 and 49\%). The treatments with $20 \%$ of TOA achieved slightly better total VFA recovery percentage than the treatments with $10 \%$. In all treatments, the best recovery was achieved from the least polar to the most polar acids (isovaleric $>$ butyric $>$ iso-butyric $>$ propionic $>$ acetic). Considering each acid individually, the treatments with 1:1 relation 

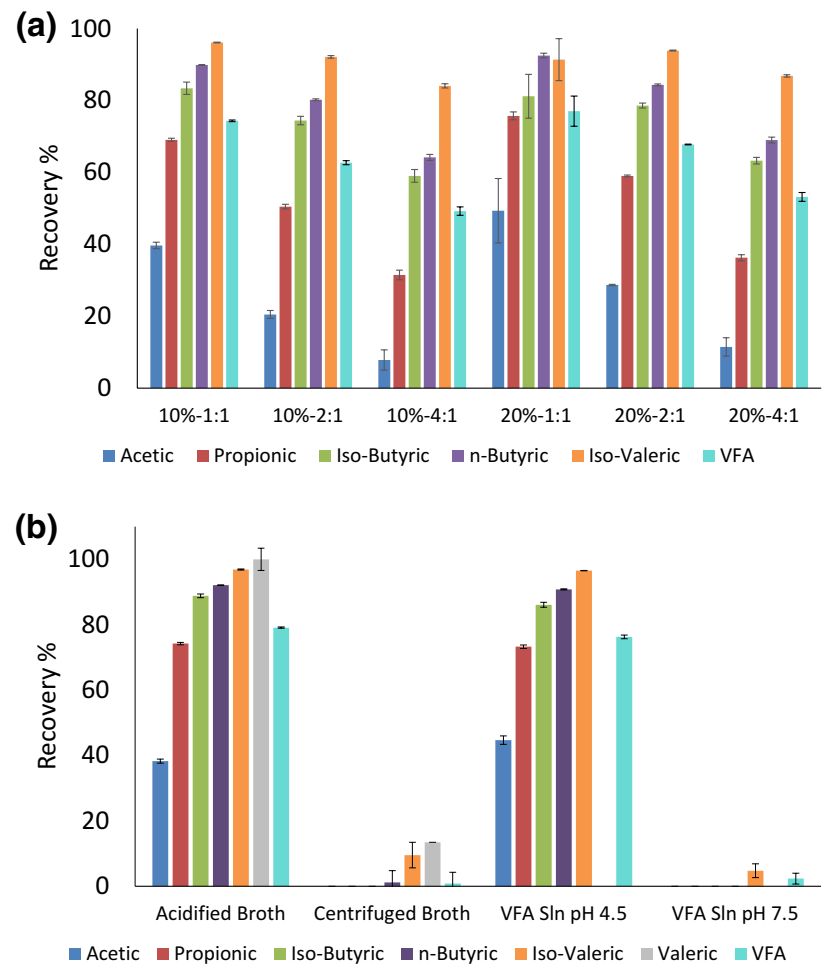

Fig. 5 a Bar plot for the VFA recovery for the experiment modifying TOA concentration, and VFA TOA/octanol relationship. b Bar plot for the VFA recovery using the slaughterhouse blood mixed fermentation broth and model solutions a regular and acidified $\mathrm{pH}$

obtained better results than the $2: 1$ and $4: 1$. The statistical analysis showed that existed significant differences between the treatments evaluated. As a factorial design, the interactions effect was evaluated using an ANOVA. The ANOVA showed the absence of interactions between the two factors (TOA concentration and VFA:TOA/octanol relation) in each individual VFA and the total VFA. The absence of interaction between the factors allowed the use of the Duncan's multiple range test to select the best treatment (Table 1). Duncan's test was performed for all the 5 acids and the total VFA. The 20\%-1:1 treatment statistically generated the best recoveries for the total VFA and all the acids except iso-valeric acid; $10 \%-1: 1$ treatment was statistically similar to 20\%-1:1 for total VFA and iso-butyric acid and was different for iso-valeric acid. Although, 10\%-1:1 and 20\%-1:1 were statistically equals for total VFA, the treatment 20\%-1:1 was selected as the best treatment. This selection is based in the ability of $20 \%-1: 1$ treatment to recover not only less polar acids as 10\%-1:1 but to achieve good recovery levels from the most polar acids.

The 20\%-1:1 treatment was used in the evaluation of the $\mathrm{pH}$ effect in model VFA solutions and slaughterhouse blood fermentation broth (Fig. 5b). Model solution (neutral $\mathrm{pH}$ ) and fermentation broth (unmodified $\mathrm{pH} /$ centrifuged) produced zero or low VFA recovery. In those treatments, the total VFA recovery was below 3\%, the less-polar acids were the acids that achieved some level of recovery, valeric $(13 \%)$, iso-valeric $(9 \%)$, and butyric $(2 \%)$. The acidified broth and the VFA model solutions at acid $\mathrm{pH}$ achieved a total VFA recovery of approximately $80 \%$. The fermentation broth achieved a slightly better recovery than the model solution treatments. This demonstrates that TOA/octanol recovery was not affected by the presence of additional compounds from the fermentation broth. The largest recoveries were observed in the less-polar acids valeric (100\%), iso-valeric (97\%), $n$-butyric (92\%), and iso-butyric (88\%). Propionic acid achieved similar values $(77 \%)$ between both, whereas, Acetic acid achieved lower recovery in the fermentation broth (38\%) than the model solution (45\%).

\section{Membrane Based Solvent Extraction Experiments}

The MBSE system (Fig. 1) was evaluated using model solutions and slaughterhouse blood fermentation broth. Figure 6 shows the VFA recovery percentage using this system. The recovery of VFA model solution $(10 \%$ solution of VFA with a composition of acetic (30\%), butyric (30\%), iso-valeric (20\%), iso-butyric (15\%) and propionic acids (5\%)) using the MBSE system for $2 \mathrm{~h}$ (Fig. 6a) achieved a Total VFA recovery of $47 \%$, this percentage was considerably lower than the achieved by the TOA/octanol solution. The acids that were less affected by the MBSE system were
Table 1 Duncan's multiple range test for the comparison of means from the recovery\% of VFA from model solutions

\begin{tabular}{lllllll}
\hline Treatment & \multicolumn{2}{l}{ Duncan grouping } \\
\cline { 2 - 7 } & Acetic acid & $\begin{array}{l}\text { Propionic } \\
\text { acid }\end{array}$ & Iso-butyric acid & $\begin{array}{l}n \text {-Butyric } \\
\text { acid }\end{array}$ & Iso-valeric acid & Total VFA \\
\hline $20 \%-1: 1$ & A & A & A & A & B & A \\
$10 \%-1: 1$ & B & B & A & B & A & A \\
$20 \%-2: 1$ & C & C & AB & C & AB & B \\
$10 \%-2: 1$ & D & D & B & D & AB & C \\
$20 \%-4: 1$ & E & E & C & E & C & D \\
$10 \%-4: 1$ & E & F & C & F & C & E \\
\hline
\end{tabular}

aTreatments with the same letter in the same column are not significantly different 

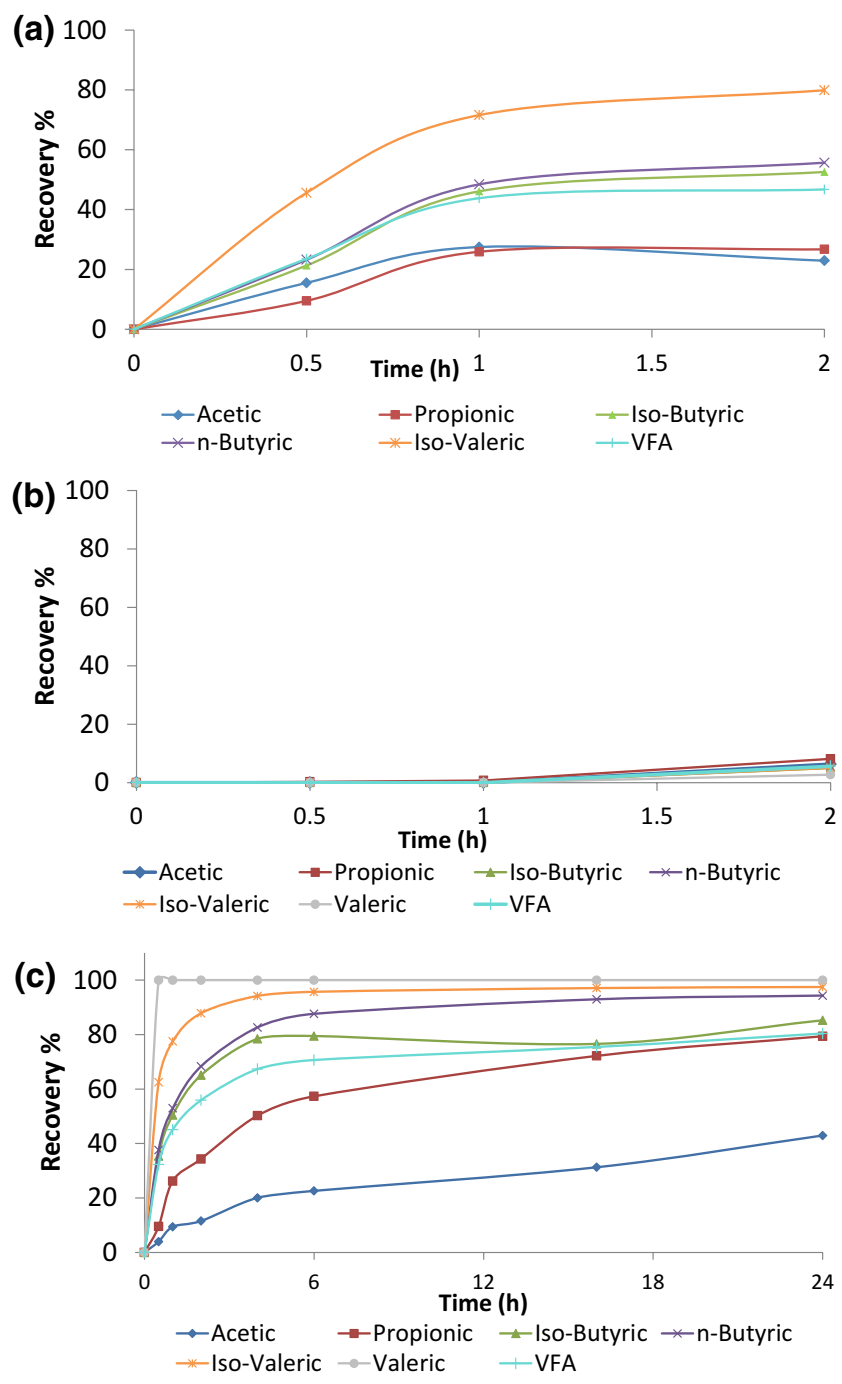

Fig. 6 VFA recovery using the MBSE system. a Model VFA solution. b Unacidified fermentation broth from slaughterhouse blood. c Acidified fermentation broth from slaughterhouse blood

iso-valeric and acetic acids, the first one achieved a recovery of $80 \%, 16 \%$ less than the TOA/octanol extraction, whereas, acetic achieved a $25 \%$ recovery, $20 \%$ less than the liquid-liquid extraction. In contrast, the other acids recovery efficiency using the MBSE system achieved a reduction between 40 and $46 \%$ versus the liquid-liquid extraction. This significant reduction in the recovery efficiency can be attributed to the membrane barrier which makes more difficult the mass transfer between liquid phases.

Un-acidified broth was used to study if it was possible to utilize the fermentation broth directly from the fermentation reactor and to observe its effect on the membrane system (Fig. 6b). As expected, the total VFA recovery using un-acidified broth was below 5\%, confirming the necessity of an acidified fermentation broth. The MBSE system was tested using acidified broth for $24 \mathrm{~h}$ (Fig. 6c).
The additional time increased the recovery at percentages comparable to the the liquid-liquid extraction experiment. In this experiment, the total VFA recovery achieved $80 \%$, individually the acids achieved values above $80 \%$ with the exception of acetic acid [valeric (100\%), iso-valeric (98\%), butyric (94\%), iso-butyric (85\%), propionic $(80 \%)$ and acetic (42\%)]. The less polar acids recovery efficiency exhibited a linear trend during the initial hours and achieved the maximum recovery at $6 \mathrm{~h}$ after that the recovery reached an equilibrium phase until the end of the process. In contrast, the recovery of acetic and propionic acid increased continuously until the end of the process. The recovery achieved by the MBSE system for model solutions and fermentation broth recorded similar values at $2 \mathrm{~h}$. The low recoveries obtained by the MBSE system using model solution was due to insufficient processing time; therefore, the extension of operating time can overcome the mass transfer resistance generated by the introduction of the membrane. Six hours appears as a reasonable time to finish the MBSE process, at this time the majority of the less polar acids achieved their maximum recovery, while the most polar acids have not achieved their maximum. The differences observed in the recovery of polar and less polar VFA can be applied as a strategy of selective recovery of VFA from slaughterhouse blood.

\section{Discussion}

In this study, esterification and MBSE methodologies were evaluated for the recovery of VFA from slaughterhouse blood fermentation broth and model VFA solutions. Esterification as a method for the recovery of carboxylic acids has been employed for the recovery of lactic, succinic and acetic acids [13, 20, 21]. However, this study is the first approximation to utilize esterification reaction for the recovery of multiple carboxylic acids and ammonium sulphate from AMF. Model solutions evidenced the effect of methanol in the esterification, in this case the increment in methanol was associated with a reduction in methyl esters yield. The methanol excess inhibited the esterification reaction by facilitating the reaction between sulphuric acid and ammonia and ammonium sulphate precipitation [13]. The reduction in available sulphuric acid reduced the esterification reaction rate by decreasing the strength of the catalyst $[13,22]$. The best methyl esters yield and ammonium sulphate production were achieved by a treatment with $80 \%$ VFA and a1:0.3:0.5 proportion of VFA: $\mathrm{H}_{2} \mathrm{SO}_{4}: \mathrm{CH}_{3} \mathrm{OH}$. VFAmethyl esters production efficiency was dependent upon the VFA concentration and affected by the existence of other materials, the late was demonstrated by the reduction between the model solutions and the fermentation 
broth. An increment in the methyl esters and ammonium sulphate production using $80 \%$ VFA instead of $50 \%$ was also found in the transformation of fermented ammonium lactate into methyl lactate and ammonium sulphate [13]. Similarly, other researchers have found that an excess of water in the reaction mixture generates equilibrium limitations [23]. Other esterification process used more diluted acid solutions (6-30\%); however, these reactions were performed using solid catalyst and/or other types of alcohols. The methyl esters yields obtained in this article were similar to the values obtained by different researchers using esterification of diluted carboxylic acids [13, $22,24]$. As expected the increment in the temperature (50 and $60^{\circ} \mathrm{C}$ ) favoured the production of methyl esters. The increment in methyl esters production via a temperature increment has been reported in other carboxylic acids esterification reactions [13]. Among VFA, the acetic acid esterification is the most studied. In this work, the production of methyl acetate from slaughterhouse blood fermentation broth achieved a yield of $64 \%$. This result corresponds with other methyl acetate yields (58 and 80\%) from diluted solutions of acetic acid [23, 25]. The 75\% yields obtained in this research for methyl propionate and butyrate were slightly lower than the maximum obtained by other studies using pure solutions [25-27]. This is the first research evaluating the recovery of iso-butyrate and iso-valerate by the production of methyl iso-butyrate and methyl iso-valerate. The esterification of those acids has been marginally studied in the production of esters with other alcohols, in those cases, their esterification yields (50\%) were lower than the reported in this research $(75$ and $83 \%)[28,29]$. The esterification reaction was more efficient for the acids with larger carbon chain; this contradicts the results obtained by Liu et al. [25], which showed that a larger carbon chain will reduce esterification reaction efficiency. This difference is created by the special characteristics in the VFA from slaughterhouse blood fermentation. The presence of water and other compounds such as untransformed blood proteins and ammonium ions are a set of conditions that have been related with a reduction in the esterification yield in acetic acid $[13,18,30]$. The polar acids can easier interact with water and the ammonium ions in the mixture inhibiting the esterification reaction; whereas, the low polarity of the long chain acids reduces their interaction with these compounds facilitating the esterification reaction. The inhibition caused by untransformed proteins can be critical in semi-continuous fermentation where the blood degradation extent is lower than batch and fed-batch reactors. Therefore, batch processes are regarded to be more suitable for esterification reaction. Methyl butyrate and methyl iso-valerate, the most produced VFA-methyl esters were, have industrial importance as scents and fragrances. In the future, the application of esterification reaction for the extraction of VFA from AFM of slaughterhouse blood need to focus in the reduction of energy costs and the increment in the esterification and ammonium sulphate yield.

MBSE systems have been studied in the recovery of butyric, propionic and acetic acid [31-33]. However, this is the first study using MBSE for the recovery of a mixture of more than two VFA and the first study using a fermentation broth from slaughterhouse blood mixed fermentation. In this study, the parameters of TOA/octanol extraction were tested by a factorial design. Acid $\mathrm{pH}$ was necessary and the ratio of $1: 1$ on a volume basis fermentation broth to TOA $(20 \%) /$ octanol $(80 \%)$ was identified. A reduction in carboxylic acids recovery efficiency with a $\mathrm{pH}$ increment was observed in the recovery of succinic and formic acid using TOA/octanol mixtures [34]. Under the optimal extraction conditions the MBSE system allowed the recovery of less polar acids such as butyric, iso-valeric and iso-butyric acids (>80\%) in favour of acetic and propionic acid (20\%). The selectivity observed in the MBSE system for less polar acids has been described in selective separation of propionic acid from a mixture of propionic and acetic acid [33]. The selectivity observed is related with hydrophobicity, solubility, and acid dissociation constant Ka. Different studies have demonstrated that VFA with greater hydrophobicity are best suited for liquid-liquid recovery [35]. The solubility of carboxylic acids is greater in mixtures of TOA/alcohol which allows an easier transfer of less polar VFA from the aqueous phase into the TOA/alcohol phase [4]. The $K_{\mathrm{a}}$ is associated with the recovery because weaker acids are more prone to be extracted from mixed solutions than strong acids [34]. In this research, acetic acid, the acid with lowest $\mathrm{p} K_{\mathrm{a}}$ (4.76), achieved the lowest recovery (25\%), whereas, the other VFA with larger $\mathrm{pKa}(4.80-4.88)$ achieved recoveries $>80 \%$. In our case, the recovery efficiency increased with the increment in the VFA carbon chain, this finding has been also reported in the extraction of VFA using liquid-liquid extraction with TOPO/kerosene $[35,36]$. The MBSE system performed at similar levels as traditional liquid-liquid extraction; however, the MBSE system has several advantages over traditional liquid-liquid extraction such as continuously VFA recovery, VFA selectivity, and solvent regeneration and reutilization. Therefore, the MBSE system is a better fit for the continuous fermentation configuration because it allows the utilization of acetic acid in recycled stream for longer-chain VFA production by creating product-induced inhibition effect. In the future, it is necessary to evaluate the effect of coupling slaughterhouse blood anaerobic mixed semi-continuous fermentation with the MBSE system and to optimize the MBSE system using an organic phase regeneration system. 


\section{Conclusions}

Esterification and MBSE achieved the recovery of the VFA produced by AMF of slaughterhouse blood. The greatest methyl esters (60-83\%) and $\left(\mathrm{NH}_{4}\right)_{2} \mathrm{SO}_{4}(50 \%)$ yields were achieved by using 80\% VFA and 1:0.3:0.5 ratio of volume for VFA: $\mathrm{H}_{2} \mathrm{SO}_{4}: \mathrm{CH}_{3} \mathrm{OH}$. The MBSE system achieved the highest VFA recovery $(>80 \%)$ using a relationship of 1:1 acidified fermentation broth to TOA $(20 \%) / o c t a n o l(80 \%)$. The esterification process is proposed to be integrated with batch processes; whereas, the MBSE system was regarded as a suitable downstream process for semi-continuous/continuous configuration. These results highlighted some essential aspects for the development of a carboxylate-platform bio-refinery from high protein wastes.

Acknowledgements The authors thank the UK Biotechnology and Biological Sciences Research Council (BBSRC) and the Anaerobic Digestion network (AD net) for funding this project through the proof of concept (PoC) funding POC2014016: 'Production and extraction of $\mathrm{C} 3$ and $\mathrm{C} 4$ aliphatic carboxylic acids from the anaerobic digestion of waste blood as a model substrate'. This work was also partially supported by EPSRC Grant: EP/I012206/1: 'Processes, mechanics and management of wastes'. Data supporting this study are openly available from the University of Southampton repository at http://doi. org/10.5258/SOTON/D0058.

Open Access This article is distributed under the terms of the Creative Commons Attribution 4.0 International License (http:// creativecommons.org/licenses/by/4.0/), which permits unrestricted use, distribution, and reproduction in any medium, provided you give appropriate credit to the original author(s) and the source, provide a link to the Creative Commons license, and indicate if changes were made.

\section{References}

1. European Comission. Horizon 2020. 2015, (2015)

2. Luengo, J.M., García, B., Sandoval, A., Naharro, G., Olivera, E.R.: Bioplastics from microorganisms. Curr. Opin. Microbiol. 6, 251-260 (2003)

3. Moita, R., Freches, A., Lemos, P.C.: Crude glycerol as feedstock for polyhydroxyalkanoates production by mixed microbial cultures. Water Res. 58, 9-20 (2014). doi:10.1016/j. watres.2014.03.066

4. Bilgin, M., Kirbaslar, S.I., Özcan, Ö, Dramur, U.: Distribution of butyric acid between water and several solvents. J. Chem. Eng. Data 51, 1546-1550 (2006)

5. Transparency Market. Carboxylic Acids (Acetic, Valeric, Isovaleric, Formic, Propionic, Butyric, Isobutyric, Citric, Caproic, Stearic, and Others) Market for Food \& Beverages, Animal Feed, Pharmaceuticals, Personal Care \& Cosmetics, Consumer Goods, Lubricants, and Other End-users-Global Industry Analysis, Size, Share, Growth, Trends and Forecast 2015-2023. september 2015, (2015)

6. Forrest, A.K., Hollister, E.B., Gentry, T.J., Wilkinson, H.H., Holtzapple, M.T.: Comparison of mixed-acid fermentations inoculated with six different mixed cultures. Bioresour. Technol. 118, 343-349 (2012). doi:10.1016/j.biortech.2012.05.043

7. Fu, Z., Holtzapple, M.T.: Consolidated bioprocessing of sugarcane bagasse and chicken manure to ammonium carboxylates by a mixed culture of marine microorganisms. Bioresour. Technol. 101, 2825-2836 (2010). doi:10.1016/j. biortech.2009.11.104

8. Forrest, A.K., Sierra, R., Holtzapple, M.T.: Effect of biodiesel glycerol type and fermentor configuration on mixed-acid fermentations. Bioresour. Technol. 101, 9185-9189 (2010). doi:10.1016/j.biortech.2010.07.041

9. Domke, S.B., Aiello-Mazzarri, C., Holtzapple, M.T.: Mixed acid fermentation of paper fines and industrial biosludge. Bioresour. Technol. 91 41-51 (2004). doi:10.1016/S0960-8524(03)00156-1

10. Placido, J., Zhang, Y.: Production and extraction of short chain carboxylic acids from the anaerobic-mixed culture fermentation of slaughterhouse blood. UK AD \& Biogas 2016, National Exhibition Centre (NEC), Birmingham, July 2016, 37-41 (2016)

11. Straathof A.J.J.: 2.57-The proportion of downstream costs in fermentative production processes. In: Moo-Young, M. (ed.) Comprehensive Biotechnology, 2nd edn., pp. 811-814. Academic Press, Burlington (2011)

12. López-Garzón, C.S., Straathof, A.J.J.: Recovery of carboxylic acids produced by fermentation. Biotechnol. Adv. 32, 873-904 (2014). doi:10.1016/j.biotechadv.2014.04.002

13. Kwak, H., Hwang, D.W., Hwang, Y.K., Chang, J.: Recovery of alkyl lactate from ammonium lactate by an advanced precipitation process. Sep. Purif. Technol. 93, 25-32 (2012). doi:10.1016/j.seppur.2012.03.025

14. Ahsan, L., Jahan, M.S., Ni, Y.: Recovery of acetic acid from the prehydrolysis liquor of kraft based dissolving pulp production process: sodium hydroxide back extraction from the trioctylamine/octanol system. Ind. Eng. Chem. Res. 52, 9270-9275 (2013)

15. Vogel, A.I.: Practical organic chemistry including qualitative organic analysis. Longmans, Green, Harlow (1964)

16. Keshav, A., Wasewar, K.L., Chand, S., Uslu, H.: Effect of binary extractants and modifier-diluents systems on equilbria of propionic acid extraction. Fluid Phase Equilib. 275, 21-26 (2009). doi:10.1016/j.fluid.2008.09.012

17. Schunk, A., Maurer, G.: On the influence of some strong electrolytes on the partitioning of acetic acid to aqueous/organic twophase systems in the presence of tri- $n$-octylamine: Part I: Methyl isobutyl ketone as organic solvent. Fluid Phase Equilib. 239, 223-239 (2006). doi:10.1016/j.fluid.2005.11.022

18. Schunk, A., Maurer, G.: On the influence of some strong electrolytes on the partitioning of acetic acid to aqueous/organic twophase systems in the presence of tri- $n$-octylamine: Part II: Toluene as organic solvent. Fluid Phase Equilib. 267, 23-38 (2008). doi:10.1016/j.fluid.2008.02.011

19. Sabolova, E., Schlosser, Š, Martak, J.: Liquid-liquid equilibria of butyric acid in water solvent systems with trioctylamine as extractant. J. Chem. Eng. Data 46, 735-745 (2001)

20. Orjuela, A., Yanez, A.J., Peereboom, L., Lira, C.T., Miller, D.J.: A novel process for recovery of fermentation-derived succinic acid. Sep. Purif. Technol. 83, 31-37 (2011). doi:10.1016/j. seppur.2011.08.010

21. Yagyu, D., Ohishi, T., Igarashi, T., Okumura, Y., Nakajo, T., Mori, Y., et al.: Recovery of acetic acid from dilute aqueous solutions using catalytic dehydrative esterification with ethanol. Chemosphere 91, 61-67 (2013). doi:10.1016/j. chemosphere.2012.11.078

22. Kasinathan, P., Hwang, D.W., Lee, U., Hwang, Y.K., Chang, J.: Effect of solvent and impurity on synthesis of ethyl lactate from fermentation-derived ammonium lactate. Chem. Eng. Sci. 66, 4549-4554 (2011). doi:10.1016/j.ces.2011.06.017 
23. Saha, B., Chopade, S.P., Mahajani, S.M.: Recovery of dilute acetic acid through esterification in a reactive distillation column. Catal. Today 60, 147-157 (2000). doi:10.1016/ S0920-5861(00)00326-6

24. Kasinathan, P., Kwak, H., Lee, U., Hwang, D.W., Hwang, Y.K., Chang, J.: Synthesis of ethyl lactate from ammonium lactate solution by coupling solvent extraction with esterification. Sep. Purif. Technol. 76, 1-7 (2010). doi:10.1016/j.seppur.2010.09.012

25. Liu, Y., Lotero, E., Goodwin, J.G. Jr.: Effect of carbon chain length on esterification of carboxylic acids with methanol using acid catalysis. J. Catal. 243, 221-228 (2006). doi:10.1016/j. jcat.2006.07.013

26. Ali, S.H.: Kinetics of catalytic esterification of propionic acid with different alcohols over Amberlyst 15. Int. J. Chem. Kinet. 41, 432-448 (2009)

27. Dange, P.N., Kulkarni, A.V., Rathod, V.K.: Ultrasound assisted synthesis of methyl butyrate using heterogeneous catalyst. Ultrason Sonochem. 26, 257-264 (2015). doi:10.1016/j. ultsonch.2015.02.014

28. Hari Krishna, S., Sattur, A.P., Karanth, N.G.: Lipae-catalyzed synthesis of isoamyl isobutyrate-optimization using a central composite rotatable design. Process Biochem. 37, 9-16 (2001). doi:10.1016/S0032-9592(01)00161-3

29. Suerbaev, K.A., Kudaibergenov, N.Z., Appazov, N., Zhaksylykova, G.Z.: Synthesis of L-menthyl isovalerate by esterification of isovaleric acid with L-menthol under microwave irradiation. Russ. J. Org. Chem. 52, 585-586 (2016)
30. Liu, Y., Lotero, E., Goodwin, J.G.: Effect of water on sulfuric acid catalyzed esterification. J. Mol. Catal. A 245, 132-140 (2006)

31. Zigová, J., Šturdík, E., Vandák, D., Schlosser, Š. Butyric acid production by Clostridium butyricum with integrated extraction and pertraction. Process Biochem. 34, 835-843 (1999). doi:10.1016/S0032-9592(99)00007-2

32. Wódzki, R., Nowaczyk, J.: Propionic and acetic acid pertraction through a multimembrane hybrid system containing TOPO or TBP. Sep. Purif. Technol. 26, 207-220 (2002). doi:10.1016/ S1383-5866(01)00164-2

33. Wódzki, R., Nowaczyk, J., Kujawski, M. Separation of propionic and acetic acid by pertraction in a multimembrane hybrid system. Sep. Purif. Technol. 21, 39-54 (2000). doi:10.1016/ S1383-5866(00)00187-8

34. Hong, Y.K., Hong, W.H., Chang, Y.K.: Effect of $\mathrm{pH}$ on the extraction characteristics of succinic and formic acids with tri-noctylamine dissolved in 1-octanol. Biotechnol. Bioprocess Eng. 6, 347-351 (2001)

35. Alkaya, E., Kaptan, S., Ozkan, L., Uludag-Demirer, S., Demirer, G.N.: Recovery of acids from anaerobic acidification broth by liquid-liquid extraction. Chemosphere 77, 1137-1142 (2009). doi:10.1016/j.chemosphere.2009.08.027

36. Yang, S.T., White, S.A., Hsu, S.T.: Extraction of carboxylic acids with tertiary and quaternary amines: Effect of $\mathrm{pH}$. Ind. Eng. Chem. Res. 30, 1335-1342 (1991) 